\title{
The Sparsest Additive Spanner via Multiple Weighted BFS Trees
}

\section{Keren Censor-Hillel}

Department of Computer Science, Technion, Haifa, Israel

ckeren@cs.technion.ac.il

\author{
Ami Paz \\ IRIF, CNRS and Paris Diderot University, Paris, France \\ amipaz@irif.fr

\section{Noam Ravid} \\ Department of Computer Science, Technion, Haifa, Israel \\ noamrvd@cs.technion.ac.il
}

\begin{abstract}
Spanners are fundamental graph structures that sparsify graphs at the cost of small stretch. In particular, in recent years, many sequential algorithms constructing additive all-pairs spanners were designed, providing very sparse small-stretch subgraphs. Remarkably, it was then shown that the known $(+6)$-spanner constructions are essentially the sparsest possible, that is, larger additive stretch cannot guarantee a sparser spanner, which brought the stretch-sparsity tradeoff to its limit. Distributed constructions of spanners are also abundant. However, for additive spanners, while there were algorithms constructing $(+2)$ and $(+4)$-all-pairs spanners, the sparsest case of $(+6)$-spanners remained elusive.

We remedy this by designing a new sequential algorithm for constructing a $(+6)$-spanner with the essentially-optimal sparsity of $\tilde{O}\left(n^{4 / 3}\right)$ edges. We then show a distributed implementation of our algorithm, answering an open problem in [10].

A main ingredient in our distributed algorithm is an efficient construction of multiple weighted BFS trees. A weighted BFS tree is a BFS tree in a weighted graph, that consists of the lightest among all shortest paths from the root to each node. We present a distributed algorithm in the CONGEST model, that constructs multiple weighted BFS trees in $|S|+D-1$ rounds, where $S$ is the set of sources and $D$ is the diameter of the network graph.
\end{abstract}

2012 ACM Subject Classification Theory of computation $\rightarrow$ Distributed computing models, Theory of computation $\rightarrow$ Sparsification and spanners, Theory of computation $\rightarrow$ Shortest paths

Keywords and phrases Distributed graph algorithms, congest model, weighted BFS trees, additive spanners

\section{Digital Object Identifier 10.4230/LIPIcs.OPODIS.2018.7}

Related Version The full version of this paper is available on the arXiv [11], https://arxiv . org/abs/1811.01997.

Funding This project has received funding from the European Union's Horizon 2020 Research And Innovation Programe under grant agreement no. 755839, and also partially supported by ISF individual research grant 1696/14. Ami Paz was supported by the Fondation Sciences Mathématiques de Paris (FSMP).

Acknowledgements We thank Shiri Chechik and Pierre Fraigniaud for discussions regarding $(+6)$-spanners. 


\section{Introduction}

A spanner of a graph $G$ is a spanning subgraph $H$ of $G$ that approximately preserves distances. Spanners find many applications in distributed computing [12, 9, 42, 43, 45], and thus their distributed construction is the center of many research papers. We focus on spanners that approximately preserve distances between all pairs of nodes, and where the stretch is only by an additive factor (purely-additive all-pairs spanners).

Out of the abundant research on distributed constructions of spanners, only two papers discuss the construction of purely additive spanners in the CONGEST model: the construction of $(+2)$-spanners is discussed in [35], and the construction of $(+4)$-spanners and $(+8)$ spanners in [10], along with other types of additive spanners and lower bounds. However, the distributed construction of (+6)-spanners remained elusive, stated explicitly as an open question in [10]. This is especially important since additive factors greater then 6 cannot yield essentially sparser spanners [2].

In this paper, we give a distributed algorithm for constructing a $(+6)$-spanner, with an optimal number of edges up to sub-polynomial factors; our spanner is even sparser than the $(+8)$-spanner presented in [10]. Several sequential algorithms building $(+6)$-spanners were presented, but none of them seems to be appropriate for a distributed setting. Thus, to achieve our result we also present a new, simple sequential algorithm for constructing $(+6)$-spanners, a result that could be of independent interest.

As a key ingredient, we provide a distributed construction of multiple weighted BFS trees. Constructing a breadth-first search (BFS) tree is a central task in many computational settings. In the classic synchronous distributed setting, constructing a BFS tree from a given source is straightforward. Due to its importance, this task has received much attention in additional distributed settings, such as the asynchronous setting (see, e.g., [40] and references therein). Moreover, at the heart of many distributed applications lies a graph structure that represents the edges of multiple BFS trees [30, 34], which are rooted at the nodes of a given subset $S \subseteq V$, where $G=(V, E)$ is the underlying communication graph. Such a structure is used in distance computation and estimation [30, 29, 34], routing table construction [34], spanner construction [10, 35, 34], and more.

When the bandwidth is limited, constructing multiple BFS trees efficiently is a non-trivial task. Indeed, distributed constructions of multiple BFS trees in the CONGEST model [40], where in each round of communication every node can send $O(\log n)$-bit messages to each of its neighbors, have been given in [30,34], who showed that it is possible to build BFS trees from a set of sources $S$ in $O(|S|+D)$ rounds, where $D$ is the diameter of the graph $G$. It is easy to show that this is asymptotically tight.

In some cases, different edges of the graph may have different attributes, which can be represented using edge weights. The existence of edge weights has been extensively studied in various tasks, such as finding or approximating lightest paths [20, 37, 27, 21, 34, 31, 4, 25], finding a minimum spanning tree (MST) in the graph [5, 23, 13], finding a maximum matching [36, 13], and more. However, as far as we are aware, no study addresses the problem of constructing multiple weighted BFS (WBFS) trees, where the goal is not to find the lightest paths from the sources to the nodes, but rather the lightest shortest paths. That is, the path in a WBFS tree from the source $s$ to a node $v$ is the lightest among all shortest paths from $s$ to $v$ in $G$.

Thus, we provide an algorithm that constructs multiple WBFS trees from a set of source nodes $S$ in the CONGEST model. Our algorithm completes in $|S|+D-1$ rounds, which implies that no overhead is needed for incorporating the existence of weights. 


\subsection{Our contribution}

At a high level, our approach for building multiple WBFS trees is to generalize the algorithm of Lenzen et al. [34] in order to handle weights. In [34], the messages are pairs consisting of a source node and a distance, which are prioritized by the distance traversed so far. When incorporating weights into this framework it makes sense to use triplets instead of pairs, where each triplet also contains the weight of the respective path. However, it may be that a node $v$ needs to send multiple messages that correspond to the same source and the same distance but contain different weights, since congestion over edges may cause the respective messages to arrive at $v$ in different rounds and, in the worst case, in a decreasing order of weights. The challenge in generalizing this framework therefore lies in guaranteeing that despite the need to consider weights, we can carefully choose a total order to prioritize triplets, such that not too many messages need to be sent, allowing us to handle congestion. Our construction and its proof appear in Section 3, giving the following.

- Theorem 1. Given a weighted graph $G=(V, E, w)$ and a set of nodes $S \subseteq V$, there exists an algorithm for the CONGEST model that constructs a WBFS tree rooted at $s$, for every $s \in S$, in $|S|+D-1$ rounds.

The importance of our multiple WBFS trees construction lies in our ability to use it for pinning down the question of constructing $(+6)$-spanners in the CONGEST model. The construction of additive spanners in the CONGEST model was studied beforehand [35, 10], but the +6 case remained unresolved, for reasons we describe below. Naturally, the quality of a spanner is measured by its sparsity, which is the motivation for allowing some stretch in the distances to begin with, and different spanners present different tradeoffs between stretch and sparsity. The properties of our $(+6)$-spanner construction algorithm are summarized in the following theorem. ${ }^{1}$

- Theorem 2. There exists an algorithm for the CONGEST model that constructs a $(+6)$ spanner with $O\left(n^{4 / 3} \log ^{4 / 3} n\right)$ edges in $O\left(\frac{n^{2 / 3}}{\log ^{1 / 3} n}+D\right)$ rounds and sucseeds w.h.p.

Previous distributed algorithms for spanners similar to ours, i.e., purely additive allpairs spanners, construct a $(+2)$-spanner with $\tilde{O}\left(n^{3 / 2}\right)$ edges in $\tilde{O}\left(n^{1 / 2}+D\right)$ rounds [35], a (+4)-spanner with $\tilde{O}\left(n^{7 / 5}\right)$ edges in $\tilde{O}\left(n^{3 / 5}+D\right)$ rounds [10], and a $(+8)$-spanner with $\tilde{O}\left(n^{15 / 11}\right)$ edges in $\tilde{O}\left(n^{7 / 11}+D\right)$ rounds [10]. Hence, our algorithm is currently the best non-trivial spanner construction algorithm in terms of density, sparser even than the previous $(+8)$-spanner. The option of getting even sparser spanners by allowing more stretch was essentially ruled out [2], while the question of improving the running time remains open for all stretch parameters.

\subsection{Other spanner construction algorithms}

Previous distributed spanner construction algorithms all build upon known sequential algorithms, and present a distributed implementations of them, or of a slight variant of them [35, 10]. For example, many sequential algorithms start in a clustering phase, where stars around high-degree nodes are added to the spanner one by one. Implementing this directly in the distributed setting will take too long; instead, it is shown that choosing cluster centers at random yields almost as good results, and can be implemented in a constant

1 We use w.h.p. to indicate a probability that is at least $1-1 / n^{c}$ for some constant $c \geq 1$ of choice. 
time. Similar methods are used for implementing other parts of the construction. However, the approach of finding a distributed implementation for a sequential algorithm fails for all known (+6)-spanner algorithms, as described next. Thus, we introduce a new sequential algorithm for the problem, and then present its distributed implementation.

There are three known approaches for the design of sequential $(+6)$-spanner algorithms. The first, presented by Baswana et al. [6], is based on measuring the quality of paths in terms of cost and value, and adding to the spanner only paths which are "affordable". This approach was later extended by Kavitha [32] to other families of additive spanners. The second approach, presented by Woodruff [46], uses a subroutine that finds almost-shortest paths between pairs of nodes, and obtains a faster algorithm at the expense of a slightly worst sparsity guarantee. The third approach, presented by Knudsen [33], is based on repeatedly going over pairs of nodes, and adding a shortest path between a pair of nodes to the spanner if their current distance in the spanner is too large.

Unfortunately, direct implementation in the CONGEST model of the known sequential algorithms is highly inefficient. We are not aware of fast distributed algorithms that allow the computation of the cost and value of paths needed for the algorithm of [6]. Similarly, for [46], the almost-shortest paths subroutine seems too costly for the CONGEST model. The algorithm of [33] needs repeated updates of the distances in the spanner between pairs of nodes after every addition of a path to it, which is a sequential process in essence, and thus we do not find it suitable for an efficient distributed implementation.

A different approach for the distributed construction of $(+6)$-spanners could be to adapt a distributed algorithm with different stretch guarantees to construct a $(+6)$-spanner This approach does not seem to work: the distributed algorithms for constructing $(+2)$ spanners [35] and (+4)-spanner [10] are both very much tailored for achieving the desired stretch, and it is not clear how to change them in order to construct sparser spanners with higher stretch. The (+8)-spanner construction algorithm [10] starts with clustering, and then constructs a (+4)-pairwise spanner between the cluster centers. Replacing the $(+4)$-pairwise spanner by a $(+2)$-pairwise spanner will indeed yield a $(+6)$-all-pairs spanner, as desired. However, even using the sparsest $(+2)$-pairwise spanners $[10,1]$, the resulting $(+6)$-spanner may have $\tilde{O}\left(n^{5 / 3}\right)$ edges, denser than our new $(+6)$-spanner and than the known $(+8)$-spanner $[10]$.

Thus, we start by presenting a new sequential algorithm for the construction of $(+6)$ spanners, an algorithm that is more suitable for a distributed implementation, and then discuss its distributed implementation. Our construction starts with a clustering phase, and then adds paths that minimize the number of additional edges that need to be added to the spanner. To implement our construction in the CONGEST model, we assign weights to the edges and use our WBFS algorithm to find shortest paths with as few edges as possible that are not yet in the spanner. Note that although the graph and the spanner we construct for it are both unweighted, the ability of our multiple WBFS algorithm to handle weights is crucial for our solution.

A (+6)-spanner must contain $n^{4 / 3} / 2^{O(\sqrt{\log n})}$ edges [2]. The best sequential algorithms [33, 6 construct a spanner with $O\left(n^{4 / 3}\right)$ edges. Our distributed algorithm constructs a spanner with $O\left(n^{4 / 3} \log ^{4 / 3} n\right)$ edges, which is slightly denser than optimal but still sparser than the $O\left(n^{4 / 3} \log ^{3} n\right)$ edges in the fast sequential construction of [46].

\subsection{Related work}

Algorithms for the CONGEST model that construct multiple (unweighted) BFS trees, rooted at a set of sources $S$, were suggested in [34] and [30], running in $O(|S|+D)$ rounds. Both algorithms start the construction of all the BFS trees simultaneously, and proceed by 
transferring messages containing the source of a BFS tree and the distance the message has traversed so far. The algorithms differ in how they order message deliveries when several messages need to be sent over an edge at the same round. We base our multiple WBFS construction on the [34] algorithm, in which messages sent by a node are prioritized by the distance they traversed so far, with a preference to messages that traversed smaller distance. The [30] algorithm, which we cannot use for our construction [28], prioritizes messages by the identity of the root, and transmits a message only in one direction of each edge in each round.

Spanners were first introduced in 1989 by [41, 42], and since then have been a topic for wide research due to their abundant applications. Prime examples for the need for sparse spanners can be found in synchronizing distributed networks [42], information dissemination [9], compact routing schemes $[12,43,45]$, and more.

Distributed constructions of various spanners have been widely studied [35, 34, 44, 10 , $6,7,14,15,16,18,19,22,17,39,24,38,26,8]$. Lower bounds were given in [44, 10, 3]. However, obtaining an efficient and sparse $(+6)$-all-pairs spanner has remained an open question [10].

Several lower bounds for the time complexity of spanner construction in the CONGEST model where presented in [10], but these are applicable only to pairwise spanners with a bounded number of pairs, and not to all-pairs spanners. A lower bound from [44] states that the construction of a spanner with $\tilde{O}\left(n^{4 / 3}\right)$ edges, such as the one we build, must take $\tilde{\Omega}\left(n^{3 / 8}\right)$ rounds. This lower bound does not take into account the bandwidth restrictions at all (it is proven for the LOCAL model), and so we believe that a higher lower bound for the CONGEST model should apply, but this is left as an intriguing open question.

\section{Preliminaries}

All graphs in this work are simple, connected and undirected. A graph can be unweighted, $G=(V, E)$, or weighted $G=(V, E, w)$ with $w: E \rightarrow\{0, \ldots, W\}$, in which case we assume $W \in \operatorname{poly}(n)$. Given a path $\rho$ in a weighted graph $G$, we use $|\rho|$ to denote the length of $\rho$, which is the number of edges in it, and $w(\rho)$ to denote the weight of the path, which is the sum of its edge-weights. The distance between two nodes $u, v$ in a graph $G$, denoted $\delta_{G}(u, v)$, is the minimum length of a path in $G$ connecting $u$ and $v$. The diameter of a graph (weighted or unweighted) is $D=\max _{u, v \in V}\left\{\delta_{G}(u, v)\right\}$.

We consider the CONGEST model of computation[40], where the nodes of a graph communicate synchronously by exchanging $O(\log n)$-bit messages along the edges. The goal is to distributively solve a problem while minimizing the number of communication rounds.

WBFS trees: We are interested in a weighted BFS tree, which consists of all lightest shortest paths from the root, formally defined as follows.

- Definition 3. Given a connected, weighted graph $G=(V, E, w)$ and a node $s \in V$, a weighted BFS tree (WBFS) for $G$ rooted at $s$ is a spanning tree $T_{s}$ of $G$ satisfying the following properties:

(i) For each $v \in V$, the path from $s$ to $v$ in $T$ is a shortest path in $G$ between $s$ and $v$.

(ii) For each $v \in V$, no shortest path from $s$ to $v$ in $G$ is lighter than the path from $s$ to $v$ in $T$.

We emphasize that this is different than requiring a subgraph containing all lightest paths from the root. One may wonder if a WBFS tree always exists, but this is easily evident by the following refinement of a (sequential) BFS search, returning a WBFS tree: go over the 
nodes in an order of non-decreasing distances from the source $s$, starting with $w(s)=0$; each node $v$ chooses as a parent a neighbor $u$ that was already processed and minimizes $w(v)=w(u)+w(u, v)$, and adds the edge $\{u, v\}$ to the tree. Each node has a single parent, so this is indeed a tree; the node ordering guarantees that this is indeed a BFS tree, assuring (i); and the parent choice guarantees the paths are lightest among the shortest, assuring (ii).

Spanners: Given a graph $G=(V, E)$, a subgraph $H=\left(V, E^{\prime}\right)$ of $G$ is called an $(\alpha, \beta)$ spanner if for every $u, v \in V$ it holds that $\delta_{H}(u, v) \leq \alpha \delta_{G}(u, v)+\beta$. The parameters $\alpha$ and $\beta$ are called the stretch parameters.

When $\alpha=1$, such a spanner is called a purely additive spanner. In this paper we focus on purely additive $(+6)$-spanners, i.e., $\alpha=1$ and $\beta=6$.

For completeness, we mention that when $\beta=0$, such a spanner is called a multiplicative spanner. In addition, while sometimes the stretch parameters need to be guaranteed only for some subset of all the pairs of nodes of the graph (such as in pairwise spanners), we emphasize that our construction provides the promise of a +6 stretch for all pairs.

\section{Multiple Weighted BFS Trees}

In the CONGEST model, the problem of finding a WBFS tree requires each node to know its parent in the WBFS tree, and the unweighted and weighted distances to the source within the tree. This allows the node to send messages to the source node through the lightest among all shortest paths. When there are multiple sources, each node should know the parent leading to each of the sources in $S$.

We define data structures for representing multiple WBFS trees. Given a node $v \in V$, the $S$-proximity-list (or proximity list for short) of $v$, noted $\mathrm{PL}_{v}^{*}$, is an ascending lexicographically ordered list of triples $(d(s, v), s, w(s, v))$, where $d(s, v)$ and $w(s, v)$ are the length and weight of the path from $s$ to $v$ in $T_{s}$. Two different triples are ordered such that $(d(s, v), s, w(s, v))<$ $(d(t, v), s, w(t, v))$ if $d(s, v)<d(t, v)$, or $d(s, v)=d(t, v)$ and $s<t$, where $s$ and $t$ may be compared by any predefined order on the node identifiers. Note that $T_{s}$ contains a single path from $s$ to $v$, so both $d(s, v)=d(t, v)$ and $s=t$ cannot happen simultaneously without having $w(s, v)=w(t, v)$.

The $S$-path-map (or path-map for short) of $v$ is a mapping from each source $s \in S$ to the parent of $v$ in $T_{s}$, noted by $\mathrm{PM}_{v}^{*}$. The list $\mathrm{PM}_{v}^{*}$ is sorted with respect to the order of $\mathrm{PL}_{v}^{*}$, such that the first records of $\mathrm{PM}_{v}^{*}$ belong to sources closest to $v$.

Algorithm 1, which constructs multiple WBFS trees from a set $S$ in the CONGEST model, is based on carefully extending the distributed Bellman-Ford-based algorithm of Lenzen et al. [34]. The heart of the algorithm is a loop (Line 7), and each iteration of it takes a single round in the CONGEST model. We show that $|S|+D-1$ iterations of the loop suffice in order to construct the desired WBSF trees.

The algorithm builds the WBFS trees by gradually updating the proximity list and the path map of each node. Each round is composed of two phases: updating the neighbors about changes in the proximity list, and receiving updates from other nodes. The path map is only used by the current node, and therefore changes to it are not sent.

Ideally, each node would update its neighbors regarding all the changes made to its proximity list. However, due to bandwidth restrictions, a node cannot send the entire list in each round. Therefore, at each round each node sends to all of its neighbors the lexicographically smallest triplet in its proximity list that it has not yet sent, while maintaining a record noting which triplets have been sent and which are waiting. Each triplet is only sent once, though a node may send multiple triplets regarding a single source. 


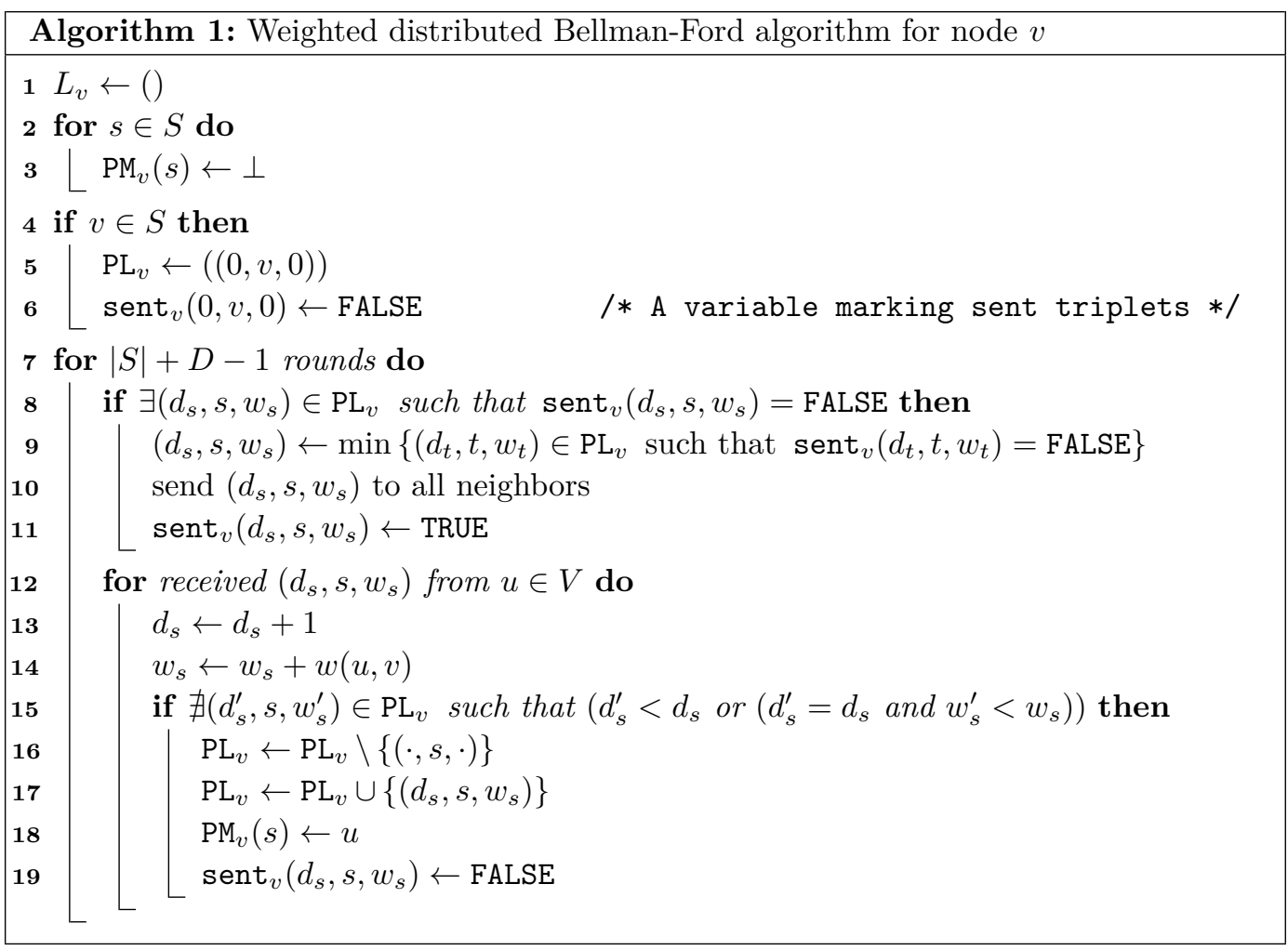

A node uses the messages received in the current round in order to update its proximity list and path map for the next round. A triplet $\left(d_{s}, s, w_{s}\right)$ received by a node $v$ from a neighbor $u$ represents the length $d_{s}$ and weight $w_{s}$ of some path $\rho$ from $s$ to $u$ in the graph. The node $v$ then considers the extended path $\rho^{\prime}=\rho \circ v$ from $s$ to $v$, compares it to its currently known best path from $s$ to $v$, and updates the proximity list and path map in case a shorter path has been found, or a lighter path with the same length.

To prove correctness, we generalize the proof of [34] to handle weights, and show that our algorithm solves the weighted $(S, d, k)$-detection problem: each node should learn which are the sources from $S$ closest to it, but at most $k$ of them and only up to distance $d$. This is formally defined as follows.

- Definition 4. Given a weighted graph $G=(V, E, w)$, a subset $S \subseteq V$ of source nodes, and a node $v \in V$, let $\mathrm{PL}_{v}^{*}$ denote the S-proximity-list and let $\mathrm{PM}_{v}^{*}$ denote the path map of the node $v$. The weighted $(S, d, k)$-detection problem requires that each node $v \in V$ learns the first $\min \left\{k, \lambda_{v}^{d}\right\}$ entries of $\mathrm{PL}_{v}^{*}$ and $\mathrm{PM}_{v}^{*}$, where $\lambda_{v}^{d}$ is the number of sources $s \in S$ such that $d(s, v) \leq d$.

Given a node $v, \mathrm{PL}_{v}$ is a variable in Algorithm 1 holding the proximity list of $v$, and we denote by $\mathrm{PL}_{v}^{(r)}$ the state of the list $\mathrm{PL}_{v}$ at the beginning of round $r$ of the algorithm, and by $\mathrm{PL}_{v}^{(\infty)}$ the value of $\mathrm{PL}_{v}$ at the end of the algorithm. Recall that $\mathrm{PL}_{v}^{*}$ is the true proximity list, so our goal is proving $\mathrm{PL}_{v}^{(\infty)}=\mathrm{PL}_{v}^{*}$, i.e., proving that the algorithm obtains the correct values of the proximity list.

We use similar notations for the path map $\mathrm{PM}_{v}^{*}$. Since the records of $\mathrm{PM}_{v}$ are updated under the same conditions as the records of $\mathrm{PL}_{v}$, the correctness of $\mathrm{PM}_{v}$ at the end of the algorithm with respect to $\mathrm{PM}_{v}^{*}$ immediately follows, and we omit the details. 
We start by showing that if there was no bound on the number of rounds, then the values of $\mathrm{PL}_{v}$ would have eventually converged to the true values of $\mathrm{PL}_{v}^{*}$. The proof of Lemma 5 can be found at the full version of this paper [11].

- Lemma 5. Given a graph $G=(V, E, w)$ and a set $S \subseteq V$, if we let the for loop in Line 7 of Algorithm 1 to run forever, then there exists a round $r_{0} \in \mathbb{N}$ such that no node $v \in V$ sends messages or modifies $\mathrm{PL}_{v}$ after round $r_{0}$. Moreover, $\mathrm{PL}_{v}^{\left(r_{0}\right)}=\mathrm{PL}_{v}^{*}$, i.e., for every $\left(d_{s}, s, w_{s}\right) \in$ $\mathrm{PL}_{v}^{\left(r_{0}\right)}$, it holds that $d_{s}=d(v, s)$ and $w_{s}=\min \left\{w(\rho) \mid \rho\right.$ connects $v$ with $s$, and $\left.|\rho|=d_{s}\right\}$.

Lemma 5 shows that without the limit on the number of rounds, the algorithm would compute the right values; however, it does not bound the number of rounds needed for this to occur. Next, we show that $|S|+D-1$ rounds suffice. We cannot apply the claims of [34] directly, since the existence of weights restricts the number of viable solutions even further, causing more updates to the proximity list and an increase in the number of messages sent. However, we do use a similar technique: we bound the number of rounds in which the $k$ smallest entries of $\mathrm{PL}_{v}$ can change.

For an entry $\left(d_{s}, s, w_{s}\right) \in \mathrm{PL}_{v}^{(r)}$, let $\ell_{v}^{(r)}\left(d_{s}, s, w_{s}\right)$ denote the index of the entry in the lexicographically ordered list $\mathrm{PL}_{v}^{(r)}$ at the beginning of round $r$. For completeness, we define $\ell_{v}^{(r)}\left(d_{s}, s, w_{s}\right)=-\infty$ if $\left(d_{s}, s, w_{s}\right)$ did not appear in $\mathrm{PL}_{v}$ at the beginning of round $r$, and $\mathrm{PL}_{v}=\infty$ if the triplet was removed from $\mathrm{PL}_{v}$ before the beginning of this round. Note that a removed triplet is never returned to the list, since the lexicographical order is transitive.

- Lemma 6. For a triplet $\left(d_{s}, s, w_{s}\right)$, the following holds:

(i) $\ell_{v}^{(r)}\left(d_{s}, s, w_{s}\right)$ is non-decreasing with $r$.

(ii) If the triplet $\left(d_{s}, s, w_{s}\right)$ is sent from a node $u$ to a node $v$ at round $r$, resulting in the addition of a new triplet $\left(d_{s}^{\prime}, s, w_{s}^{\prime}\right)$ to $\mathrm{PL}_{v}$ at the end of round $r$, where $d_{s}^{\prime}=d_{s}+1$ and $w_{s}^{\prime}=w_{s}+w(u, v)$, then $\ell_{u}^{(r)}\left(d_{s}, s, w_{s}\right) \leq \ell_{v}^{(r+1)}\left(d_{s}^{\prime}, s, w_{s}^{\prime}\right)$.

Part (i) follows from the fact that the number of triplets below $\left(d_{s}, s, w_{s}\right)$ cannot decrease. To prove part (ii), we show that all the triplets below $\left(d_{s}, s, w_{s}\right)$ in $\mathrm{PL}_{u}$ are sent from $u$ to $v$ and added to $\mathrm{PL}_{v}$ before $\left(d_{s}, s, w_{s}\right)$ is sent and added.

Proof. Part (i) is a consequence of the method used by our algorithm for managing the list $\mathrm{PL}_{v}$. According to our algorithm, triplets are not removed from $\mathrm{PL}_{v}$ when they are sent. The only case in which a triplet $\left(d_{t}, t, w_{t}\right)$ is removed from $\mathrm{PL}_{v}$ is when a lexicographically smaller triplet $\left(d_{t}^{\prime}, t, w_{t}^{\prime}\right)$ is added to the list instead. When this happens in round $r$, it holds that $\ell_{v}^{(r)}\left(d_{t}, t, w_{t}\right) \geq \ell_{v}^{(r+1)}\left(d_{t}^{\prime}, t, w_{t}^{\prime}\right)$, since the new triplet is lexicographically smaller. Hence, for every other triplet $\left(d_{s}, s, w_{s}\right) \in \mathrm{PL}_{v}$, the number of lexicographically smaller triplets in $\mathrm{PL}_{v}$ cannot decrease throughout the algorithm.

We now turn to prove part (ii) of the lemma. By the fact that the triplet $\left(d_{s}, s, w_{s}\right)$ is sent by the node $u$ in round $r$, we conclude that the $\ell_{u}^{(r)}\left(d_{s}, s, w_{s}\right)-1$ triplets preceding it in the list $\mathrm{PL}_{u}^{(r)}$ have already been sent by $u$ in earlier rounds, and arrived at the node $v$. For each such triplet $\left(d_{t}, t, w_{t}\right)$, either $d_{t} \leq d_{s}$, or $t<s$ and $d_{t}=d_{s}$. Therefore, when added to $\mathrm{PL}_{v}$ as $\left(d_{t}+1, t, w_{t}+w(u, v)\right)$ it is lexicographically smaller than $\left(d_{s}^{\prime}, s, w_{s}^{\prime}\right)$. At round $r$, either $\left(d_{t}+1, t, w_{t}+w(u, v)\right)$ is in $\mathrm{PL}_{v}^{(r)}$ or it was replaced by a lexicographically smaller triplet containing $t$. Thus, there are at least $\ell_{u}^{(r)}\left(d_{s}, s, w_{s}\right)-1$ triplets smaller than $\left(d_{s}^{\prime}, s, w_{s}^{\prime}\right)$ in $\mathrm{PL}_{v}^{(r+1)}$, and hence $\ell_{u}^{(r)}\left(d_{s}, s, w_{s}\right) \leq \ell_{v}^{(r+1)}\left(d_{s}^{\prime}, s, w_{s}^{\prime}\right)$.

Lemma 6 implies that as the algorithm progresses, messages at higher indexes of the proximity list are sent and updated. This can be used to obtain an upper bound on the round in which a triplet at a certain index of the proximity list can be sent or received, as formalized by the next lemma. 
Lemma 7. In round $r \in \mathbb{N}$ of Algorithm 1, a node $v \in V$ can:

(i) send a message $\left(d_{s}, s, w_{s}\right)$ only if

$$
d_{s}+\ell_{v}^{(r)}\left(d_{s}, s, w_{s}\right) \geq r
$$

(ii) add to $\mathrm{PL}_{v}$ a triplet $\left(d_{s}, s, w_{s}\right)$ only if

$$
d_{s}+\ell_{v}^{(r+1)}\left(d_{s}, s, w_{s}\right)>r
$$

Part (i), when put in words, is rather intuitive: while a triplet might need to wait before being sent, the waiting time is bounded from above by the distance the triplet has traversed from its source, plus the number of triplets that were to be sent before it. Part (ii) is complementary to part (i): the time before a triplet is added, is, once more, bounded by the distance it traversed plus the number of lexicographically smaller triplets.

Proof. We start by showing that, for a given round $r$, if Lemma 7(i) holds for all nodes then Lemma 7 (ii) holds as well. Consider a triplet $\left(d_{s}^{\prime}, s, w_{s}^{\prime}\right)$ that is added to $\mathrm{PL}_{v}$ as a result of a message $\left(d_{s}, s, w_{s}\right)$ sent from $u$ to $v$ in round $r$, where $d_{s}^{\prime}=d_{s}+1$ and $w_{s}^{\prime}=w_{s}+w(u, v)$. Lemma $7(\mathrm{i})$ implies that $d_{s}+\ell_{u}^{(r)}\left(d_{s}, s, w_{s}\right) \geq r$, and by Lemma 6(ii) we have that $\ell_{v}^{(r+1)}\left(d_{s}^{\prime}, s, w_{s}^{\prime}\right) \geq \ell_{u}^{(r)}\left(d_{s}, s, w_{s}\right)$. As $d_{s}^{\prime}>d_{s}$, we conclude

$$
d_{s}^{\prime}+\ell_{v}^{(r+1)}\left(d_{s}^{\prime}, s, w_{s}^{\prime}\right)>d_{s}+\ell_{u}^{(r)}\left(d_{s}, s, w_{s}\right) \geq r
$$

which implies Lemma 7 (ii).

Next, we prove by induction that both parts of the lemma hold. In round 1, Lemma 7(i) holds trivially, since by definition $\ell_{v}^{(1)}\left(d_{s}, s, w_{s}\right) \geq 1$. Assume that Lemma 7 holds at round $r-1$; we show the lemma holds at round $r$. Since Lemma 7(i) implies Lemma 7(ii), it is sufficient to show that every message $\left(d_{s}, s, w_{s}\right)$ sent by some node $v \in V$ in round $r$ satisfies $d_{s}+\ell_{v}^{(r)}\left(d_{s}, s, w_{s}\right) \geq r$.

Observe that if $\left(d_{s}, s, w_{s}\right)$ is sent by a node $v$ in round $r$, then the triplet must have been added to $\mathrm{PL}_{v}$ in some round $r^{\prime} \leq r-1$. If $r^{\prime}=r-1$, according to the induction hypothesis, Lemma $7\left(\right.$ ii) holds and $d_{s}+\ell_{v}^{(r)}\left(d_{s}, s, w_{s}\right)>r-1$, implying $d_{s}+\ell_{v}^{(r)}\left(d_{s}, s, w_{s}\right) \geq r$, since all the terms are integers.

Otherwise $r^{\prime}<r-1$. In this case, in round $r-1$ the triplet $\left(d_{s}, s, w_{s}\right)$ appeared in $\mathrm{PL}_{v}$ and was not yet sent. Since $\left(d_{s}, s, w_{s}\right)$ is sent in round $r$, a different triplet $\left(d_{t}, t, w_{t}\right)$ with $t \neq s$ must have been sent in round $r-1$, implying:

$$
d_{s}+\ell_{v}^{(r-1)}\left(d_{s}, s, w_{s}\right)>d_{t}+\ell_{v}^{(r-1)}\left(d_{t}, t, w_{t}\right) .
$$

By Lemma 6 (i), we have that $\ell_{v}^{(r)}\left(d_{s}, s, w_{s}\right) \geq \ell_{v}^{(r-1)}\left(d_{s}, s, w_{s}\right)$, and combined with the induction hypothesis for Lemma $7(\mathrm{i})$ in round $r-1$ we conclude:

$$
d_{s}+\ell_{v}^{(r)}\left(d_{s}, s, w_{s}\right) \geq d_{s}+\ell_{v}^{(r-1)}\left(d_{s}, s, w_{s}\right)>d_{t}+\ell_{v}^{(r-1)}\left(d_{t}, t, w_{t}\right) \geq r-1 .
$$

This gives that $d_{s}+\ell_{v}^{(r)}\left(d_{s}, s, w_{s}\right) \geq r$, since all the terms are integers.

Lemma 5 implies that eventually, the lists $\mathrm{PL}_{v}$ converge to contain the correct values, and Lemma 7 restricts the number of rounds in which specific list entries may change. From this, we conclude that the algorithm solves the weighted $(S, d, k)$-detection problem.

- Lemma 8. Given an instance of the weighted $(S, d, k)$-detection problem, for every $v \in V$ and round $r$ of an execution of Algorithm 1 with

$$
r \geq \min \{d, D\}+\min \{k,|S|\}
$$


the truncation of $\mathrm{PL}_{v}^{(r)}$ to the first $\min \left\{k, \lambda_{v}^{d}\right\}$ entries, where $\lambda_{v}^{d}$ is the number sources $s \in S$ such that $d(s, v) \leq d$, solves weighted $(S, d, k)$-detection problem.

This lemma says that the truncated list is correct at the beginning of the relevant round. To prove it, we use Lemma 7 (ii) to show that the values in the truncated list cannot change at round $r$ or later, and Lemma 5 to deduce they are correct.

Proof. Assume w.l.o.g that $d \leq D$, as $D$ bounds the distance to any source, and $k \leq|S|$, as otherwise $v$ needs to learn about all sources.

By Lemma 5 , there is a round $r_{0}$ when all entries of $\mathrm{PL}_{v}^{\left(r_{0}\right)}$ are correct, and let $\left(d_{s}, s, w_{s}\right)$ be a triplet in one of the first $\min \left\{k, \lambda_{v}^{d}\right\}$ entries of $\mathrm{PL}_{v}^{\left(r_{0}\right)}$. Since $\left(d_{s}, s, w_{s}\right)$ is one of the first $\lambda_{v}^{d}$ entries and $\mathrm{PL}_{v}^{\left(r_{0}\right)}=\mathrm{PL}_{v}^{*}$, we have $d_{s} \leq d$.

Let $r$ be the round when $\left(d_{s}, s, w_{s}\right)$ is inserted to the list $\mathrm{PL}_{v}$. By Lemma $7(\mathrm{ii}), r<$ $d_{s}+\ell_{v}^{(r+1)}\left(d_{s}, s, w_{s}\right)$. By Lemma 6(i), when the triplet is inserted to the list, it is already placed in one of the first $\min \left\{k, \lambda_{v}^{d}\right\}$ entries, i.e., $\ell_{v}^{(r+1)}\left(d_{s}, s, w_{s}\right) \leq \min \left\{k, \lambda_{v}^{d}\right\} \leq k$. Hence,

$$
r<d_{s}+\ell_{v}^{(r+1)}\left(d_{s}, s, w_{s}\right) \leq d+k
$$

Since this claim holds for any of the first $\min \left\{k, \lambda_{v}^{d}\right\}$ entries, these were all correct at the beginning of round $d+k$, and in all the succeeding rounds.

The construction of multiple WBFS trees is an instance of the $(S, D,|S|)$-detection problem. Lemma 8 shows that after $|S|+D-1$ rounds of Algorithm 1 on such an instance, all the entries of the list $\mathrm{PL}_{v}^{(|S|+D)}$ are correct, yielding the main result of this section.

Theorem 1 (restated). Given a weighted graph $G=(V, E, w)$ and a set of nodes $S \subseteq V$, there exists an algorithm for the CONGEST model that constructs a WBFS tree rooted at $s$, for every $s \in S$, in $|S|+D-1$ rounds.

\section{A (+6)-Spanner Construction}

In this section we discuss the distributed construction of $(+6)$-spanners. First, we present a template for constructing a $(+6)$-spanner and analyze the stretch and sparsity of the constructed spanner. Then, we provide an implementation of our template in the CONGEST model and analyze its running time.

A cluster $C_{i}$ around a cluster center $c_{i} \in V$ is a subset of the set of neighbors of $c_{i}$ in $G$. A node belonging to a cluster is clustered, while the other nodes are unclustered.

Our algorithm starts by randomly choosing cluster centers, and adding edges between them to their neighbors, where each neighbor arbitrarily chooses a single center to connect to. Then, additional edges are added, to connect each unclustered node to all its neighbors. Next, shortest paths between clusters are added to the spanner. In order to find these shortest paths in the CONGEST model, we use the WBFS construction algorithm to build WBFS trees from random sources. At the heart of our algorithm stands the path-hitting framework of Woodruff [46]: a shortest path in the graph which has many edges between clustered nodes, must go through many clusters. This fact is used in order to show that a path with many missing edges (edges not in $H$ ) is more likely to have an adjacent source of a WBFS tree, and thus it is well approximated by a path within the spanner.

Woodruff's algorithm starts with a similar clustering step. However, in order to add paths between clusters, it uses an involved subroutine that finds light almost-shortest paths between pairs of nodes. This subroutine seems too global to be implemented efficiently in a 
distributed setting, so in our construction it is replaced by only considering lightest shortest paths, which we do using the WBFS trees defined earlier.

Our algorithm constructs a $(+6)$-spanner with $O\left(n^{4 / 3} \log ^{4 / 3} n\right)$ edges in $\tilde{O}\left(n^{2 / 3}+D\right)$ rounds, as stated next.

Theorem 2 (restated). There exists an algorithm for the CONGEST model that constructs a $(+6)$-spanner with $O\left(n^{4 / 3} \log ^{4 / 3} n\right)$ edges in $O\left(\frac{n^{2 / 3}}{\log ^{1 / 3} n}+D\right)$ rounds and sucseeds w.h.p.

Lemmas 9 and 10 analyze the size and stretch of Algorithm 6AP given below. The number of rounds of its distributed implementation is analyzed in Lemma 11, giving Theorem 2. We use $c>2$ to denote a constant that can be chosen according to the desired exponent of $1 / n$ in the failure probability.

\section{Algorithm 6AP}

Input: a graph $G=(V, E)$, a constant $c>2$;

Output: a subgraph $H$ of $G$;

Initialization: $n \leftarrow|V| ; H \leftarrow(V, \emptyset) ; k \leftarrow 1$

Clustering. Pick each node as a cluster center w.p. $\frac{c}{n^{1 / 3} \log ^{1 / 3} n}$, and denote the set of selected nodes by $\mathcal{C}=\left\{c_{1}, c_{2}, \ldots\right\}$. For each $c_{i}$, initialize a cluster $C_{i} \leftarrow \emptyset$.

For each node $v \in V$, choose a neighbor $c_{i}$ of $v$ which is a cluster center, if such a neighbor exists, add the edge $\left(v, c_{i}\right)$ to $H$, and add $v$ to $C_{i}$. If none of the neighbors of $v$ is a cluster center, add to $H$ all the edges adjacent to $v$. Let $H_{0} \leftarrow H$.

\section{Path Buying.}

While $k \leq \frac{8 c n^{2 / 3}}{\log ^{1 / 3} n}$ do:

1. $S_{k} \leftarrow \emptyset$

2. Add each cluster center $c_{i} \in \mathcal{C}$ to $S_{k}$ w.p. $\frac{8 c^{2} \log n}{k}$, independently of the other centers

3. For each pair $\left(c_{i}, c_{j}\right) \in \mathcal{C} \times S_{k}$ :
a. $A \leftarrow \emptyset$
$/^{*} A$ is a set of paths */

b. For each $v \in C_{j}$ :

i. Among all the shortest paths from $c_{i}$ to $v$, let $P_{v}$ be a path with minimum $\left|P_{v} \backslash H_{0}\right|$

ii. If $\left|P_{v} \backslash H_{0}\right|<2 k$, add $P_{v}$ to $A$

c. If $A \neq \emptyset$, add to $H$ one of the shortest among the paths of $A$

4. $k \leftarrow 2 k$

- Lemma 9. Algorithm 6AP outputs a subgraph $H$ of $G$ with $O\left(n^{4 / 3} \log ^{4 / 3} n\right)$ edges, with probability at least $1-O\left(n^{-c+1}\right)$.

Proof. The algorithm starts with $H=(V, \emptyset)$ and only adds edges from $G$, so $H$ is indeed a subgraph of $G$ over the same node set.

In the first part of the clustering phase, each node adds to $H$ at most one edge, connecting it to a single cluster center, for a total of $O(n)$ edges. Then, the probability that a node of degree at least $n^{1 / 3} \log ^{4 / 3} n$ is left unclustered is at most $\left(1-\frac{c}{n^{1 / 3} \log ^{1 / 3} n}\right)^{n^{1 / 3} \log ^{4 / 3} n}$, which is $O\left(n^{-c}\right)$. A union bound implies that all nodes of degree at least $n^{1 / 3} \log ^{4 / 3} n$ are clustered w.p. $1-O\left(n^{-c+1}\right)$, and thus the total number of edges added to $H$ by unclustered nodes in the second part of the clustering phase is $O\left(n^{4 / 3} \log ^{4 / 3} n\right)$, w.p. $1-O\left(n^{-c+1}\right)$. 


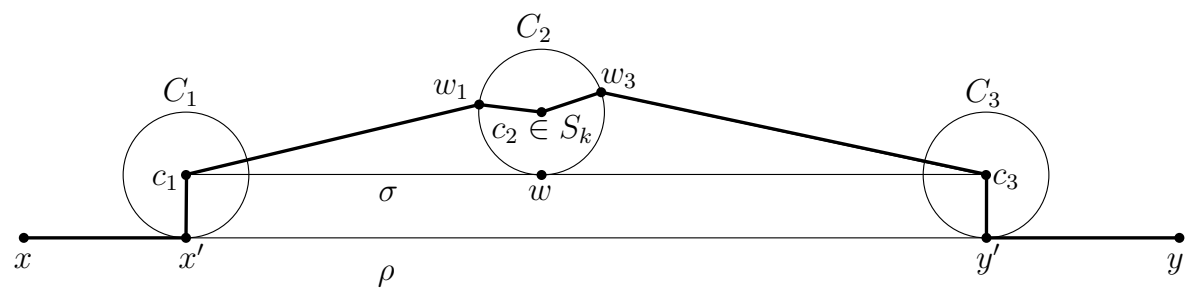

Figure 1 Illustration of the proof of Lemma 10

We start the analysis of the path buying phase by bounding the size of $\mathcal{C}$. A node $v \in V$ is added to $\mathcal{C}$ w.p. $\frac{c}{n^{1 / 3} \log ^{1 / 3} n}$, so $\mathbb{E}[|\mathcal{C}|]=\frac{c n^{2 / 3}}{\log ^{1 / 3} n}$. A Chernoff bound implies that

$$
\operatorname{Pr}\left[|\mathcal{C}|>\frac{4 c n^{2 / 3}}{\log ^{1 / 3} n}\right] \leq \exp \left(-\frac{c n^{2 / 3}}{\log ^{1 / 3} n}\right)=o\left(n^{-c}\right)
$$

Similarly, for each value of $k$, we have $\mathbb{E}\left[\left|S_{k}\right|\right]=\frac{8 c^{2} n^{2 / 3} \log ^{2 / 3} n}{k}$, and

$$
\operatorname{Pr}\left[\left|S_{k}\right|>\frac{32 c^{2} n^{2 / 3} \log ^{2 / 3} n}{k}\right] \leq \exp \left(-\frac{8 c^{2} n^{2 / 3} \log ^{2 / 3} n}{k}\right)=O\left(n^{-c}\right),
$$

where the last equality follows since $k \leq \frac{n^{2 / 3}}{\log ^{1 / 3} n}$. A union bound implies that $|\mathcal{C}|=$ $O\left(\frac{n^{2 / 3}}{\log ^{1 / 3} n}\right)$ and $\left|S_{k}\right|=O\left(\frac{n^{2 / 3} \log ^{2 / 3} n}{k}\right)$ for all $k$, w.p. at least $1-O\left(n^{-c+1}\right)$.

Finally, for each $k$, for each $\left(c_{i}, c_{j}\right) \in \mathcal{C} \times S_{k}$ we add at most one path with less than $2 k$ missing edges to $H$. Thus, for each value of $k$ we add less than $|\mathcal{C}| \cdot\left|S_{k}\right| \cdot 2 k=O\left(n^{4 / 3} \log ^{1 / 3} n\right)$ edges to $H$, w.p. at least $1-O\left(n^{-c+1}\right)$. Summing over all $O(\log n)$ values of $k$, and adding the number of edges contributed by the clustering phase, we conclude that $H$ has at most $O\left(n^{4 / 3} \log ^{4 / 3} n\right)$ edges, w.p. at least $1-O\left(n^{-c+1}\right)$.

- Lemma 10. The graph $H$ constructed by Algorithm 6AP satisfies $\delta_{H}(x, y) \leq \delta_{G}(x, y)+6$ for each pair $(x, y) \in V \times V$, with probability at least $1-O\left(n^{-c+2}\right)$.

Proof. Consider a shortest path $\rho$ in $G$ between two nodes $x, y \in V$ (see Figure 1). Let $x^{\prime}$ and $y^{\prime}$ be the first and last clustered nodes on $\rho$, respectively. If all nodes of $\rho$ are unclustered, then $\rho$ is fully contained in $H_{0}$ and we are done.

Let $c_{1}$ and $c_{3}$ be the centers of the clusters containing $x^{\prime}$ and $y^{\prime}$, respectively. Let $\sigma$ be a shortest path in $G$ between $c_{1}$ and $c_{3}$, and denote by $k^{\prime}$ the number of edges of $\sigma \backslash H_{0}$. Let $k$ be the largest power of 2 such that $k \leq k^{\prime}$.

An edge can be in $\sigma \backslash H_{0}$ only if it connects two clustered nodes. Hence, $k^{\prime}$, the number of edges in $\sigma \backslash H_{0}$, is smaller than the number of clustered nodes in $\sigma$. On the other hand, $\sigma$ cannot contain more than three nodes of the same cluster: the distance between every two nodes in a cluster is at most two, so a shortest path cannot traverse more than three nodes of the same cluster. Thus, the number of clusters intersecting $\sigma$ is at least $k^{\prime} / 3$. As $k^{\prime} / 3 \geq k / 3$, the probability that none of the centers of these clusters is chosen to $S_{k}$ is at most $\left(1-\frac{8 c^{2} \log n}{k}\right)^{k / 3}=O\left(n^{-c^{2}}\right)$. For each pair of nodes, a cluster center on a shortest path between them is chosen to $S_{k}$, for the appropriate value of $k$, with similar probability. A union bound implies that this claim holds for all pairs in $V \times V$ w.p. at least $1-O\left(n^{-c^{2}+2}\right)$. 
Let $w$ be a node on $\sigma$ in a cluster $C_{2}$ such that $c_{2} \in S_{k}$, if such a cluster exists. Denote by $\sigma\left[c_{1}, w\right]$ the sub-path of $\sigma$ from $c_{1}$ to $w$. As there are $k^{\prime}<2 k$ edges in $\sigma \backslash H_{0}$, there are also less than $2 k$ edges in $\sigma\left[c_{1}, w\right] \backslash H_{0}$. Thus, in step $3(b)$ of the path-buying phase for $k$, either the path $\sigma\left[c_{1}, w\right]$ or some other path between $c_{1}$ and $w$ of length at most $\delta_{G}\left(c_{1}, w\right)$ is added to $A$. In step $3(c)$, a path from $c_{1}$ to some node $w_{1} \in C_{2}$ is added to $H$, and this is a shortest path in $A$, so $\delta_{H}\left(c_{1}, w_{1}\right) \leq \delta_{G}\left(c_{1}, w\right)$. Similarly, a shortest path from $c_{3}$ to some $w_{3} \in C_{2}$ is added to $H$, and $\delta_{H}\left(c_{3}, w_{3}\right) \leq \delta_{G}\left(c_{3}, w\right)$.

The path $\sigma$ is a shortest path from $c_{1}$ to $c_{3}$ in $G$, so $|\sigma| \leq \delta_{G}\left(x^{\prime}, y^{\prime}\right)+2$. As $\delta_{G}\left(c_{1}, w\right)+$ $\delta_{G}\left(c_{3}, w\right)=|\sigma|$, we conclude $\delta_{H}\left(c_{1}, w_{1}\right)+\delta_{H}\left(c_{3}, w_{3}\right) \leq|\sigma| \leq \delta_{G}\left(x^{\prime}, y^{\prime}\right)+2$.

Consider the path from $x$ to $y$ in $H$ composed of the sub-path of $\rho$ from $x$ to $x^{\prime}$, the edge $\left(x^{\prime}, c_{1}\right)$, the path from $c_{1}$ to $w_{1}$, the edges $\left(w_{1}, c_{2}\right)$ and $\left(c_{2}, w_{3}\right)$, the path from $w_{3}$ to $c_{3}$, the edge $\left(c_{3}, y^{\prime}\right)$, and finally, the sub-path of $\rho$ from $y^{\prime}$ to $y$. This is a path from $x$ to $y$ in $H$, implying

$$
\begin{aligned}
\delta_{H}(x, y) & \leq \delta_{H}\left(x, x^{\prime}\right)+1+\delta_{H}\left(c_{1}, w_{1}\right)+2+\delta_{H}\left(w_{3}, c_{3}\right)+1+\delta_{H}\left(y^{\prime}, y\right) \\
& \leq \delta_{G}\left(x, x^{\prime}\right)+4+\delta_{G}\left(x^{\prime}, y^{\prime}\right)+2+\delta_{G}\left(y^{\prime}, y\right)=\delta_{G}(x, y)+6,
\end{aligned}
$$

as desired.

We now discuss the implementation of Algorithm 6AP in the CONGEST model.

- Lemma 11. Algorithm 6AP can be implemented in $O\left(\frac{n^{2 / 3}}{\log ^{1 / 3} n}+D\right)$ rounds in the CONGEST model, with probability at least $1-o\left(n^{-c}\right)$.

Proof. For the clustering phase, each node decides locally w.p. $\frac{c}{n^{1 / 3} \log ^{1 / 3} n}$ to become a cluster center, and notifies its neighbors. Each node with a neighbor that is a cluster center now joins a cluster by sending a message to such a neighbor and adding the appropreate edge to the spanner. A node with no neighboring cluster centers notifies all its neighbors and adds all its edges to the spanner. This is done in a constant number of rounds.

Before the path buying phase, the nodes construct a single BFS tree, along which they compute an upper bound $D^{\prime}$ on $D$, satisfying $D \leq D^{\prime}<2 D$, and count the number of cluster centers, $|\mathcal{C}|$. The nodes mark the edges of $H_{0}$ with weight 0 and the other edges with weight 1. Then, they construct a WBFS tree rooted at each cluster center by executing Algorithm 1 for $|\mathcal{C}|+D^{\prime}$ many rounds. By the proof of Lemma 9, we have $|\mathcal{C}| \in O\left(\frac{n^{2 / 3}}{\log ^{1 / 3} n}\right)$ w.p. at least $1-o\left(n^{-c}\right)$, and thus the construction of the WBFS trees takes $O\left(\frac{n^{2 / 3}}{\log ^{1 / 3} n}+D\right)$ rounds with the same probability.

Each node $v$ now knows about a "good" path to each cluster center $c_{i}$, i.e., a shortest path from $c_{i}$ to $v$, with a minimal number of edges not in $H$ after the clustering phase. A node $v$ in a cluster $C_{j}$ notifies its neighbor $c_{j}$ about all the distances to other cluster centers in $\mathcal{C}$ and the number of missing edges in each such path. That is, each $v \in C_{j}$ sends $|\mathcal{C}|$ messages to $c_{j}$, which takes $O\left(\frac{n^{2 / 3}}{\log ^{1 / 3} n}\right)$ rounds.

Each cluster center $c_{j}$ decides locally to join each set $S_{k}$ w.p. $\frac{8 c^{2} \log n}{k}$. For each other center $c_{i} \in \mathcal{C}, c_{j}$ locally constructs the list $A$ : for each $v \in C_{j}, A$ contains the shortest path from $c_{i}$ to $v \in C_{j}$ found by the WBFS algorithm, and the number of missing edges in it. Then, $c_{j}$ chooses from $A$ a path from $c_{i}$ to some $v \in C_{j}$ with a minimal number of missing edges, and if it has at most $2 k$ missing edges, $c_{j}$ sends a "buy $c_{i}$ " message to $v$.

Finally, all nodes simultaneously execute a "buy" phase, where "buy $c_{i}$ " messages are sent up the WBFS tree. To avoid congestion, we assume that during the execution of Algorithm 1, each node keeps a record of the messages it got in each round and the WBFS source each 
message referred to. Each node $v$ then sends messages in reversed order: if $v$ has a message "buy $c_{i}$ ", and it got a message from $u$ regarding $c_{i}$ in the $r$-before-last round of Algorithm 1 , then it sends the message "buy $c_{i}$ " to $u$ in round $r$ of the "buy" phase. Then, $u$ adds "buy $c_{i}$ " to its list of messages, and adds the edge $(u, v)$ to the spanner. This parts takes $O\left(\frac{n^{2 / 3}}{\log ^{1 / 3} n}+D\right)$ rounds, just like the execution of Algorithm 1.

\section{$5 \quad$ Discussion and Open Questions}

While we present an application of WBFS trees, our algorithm also solves the weighted $(S, d, k)$-detection problem, a result the could be of independent interest.

The question of finding the lightest paths between all pairs of nodes in a graph is a fundamental question in many computational models. In the CONGEST model, no exact algorithm for the problem running in $O(n)$ rounds is known. This major question is still left open here, but we hope our study of lightest shortest paths could facilitate future research on the question of finding lightest paths.

While this paper settles the question of constructing sparse (+6)-spanners fast, the study of spanner construction in distributed environments still lags behind the study of sequential spanner construction algorithms. In the field of purely additive spanners, we still do not have fast algorithms, e.g., for the construction of sparse $(+0)$-pairwise spanners (a.k.a. pairwise preservers) and (+6)-pairwise spanners.

A more intriguing question is proving time lower bounds for the construction of spanners in the CONGEST model: while $\Omega(D)$ rounds are known to be necessary [44], lower bounds that depend on other parameters of the graph or the spanners exist only for pairwise spanners [10]. Finding a lower bound for the construction of all-pairs spanners in the CONGEST model is still an open question. Such a lower bound could show that the $n^{3 / 2}$ term in the time bound of our construction is inevitable, or motivate a design of faster algorithms for the problem.

\section{References}

1 Amir Abboud and Greg Bodwin. Error Amplification for Pairwise Spanner Lower Bounds. In 27th Annual ACM-SIAM Symposium on Discrete Algorithms, SODA, pages 841-854, 2016.

2 Amir Abboud and Greg Bodwin. The 4/3 Additive Spanner Exponent Is Tight. J. ACM, 64(4):28:1-28:20, 2017.

3 Amir Abboud, Keren Censor-Hillel, and Seri Khoury. Near-Linear Lower Bounds for Distributed Distance Computations, Even in Sparse Networks. In 30th International Symposium on Distributed Computing, DISC, pages 29-42, 2016.

4 Udit Agarwal, Vijaya Ramachandran, Valerie King, and Matteo Pontecorvi. A Deterministic Distributed Algorithm for Exact Weighted All-Pairs Shortest Paths in Õ $\left(n^{3 / 2}\right)$ Rounds. In ACM Symposium on Principles of Distributed Computing, PODC, pages 199-205, 2018.

5 Baruch Awerbuch. Optimal Distributed Algorithms for Minimum Weight Spanning Tree, Counting, Leader Election and Related Problems. In ACM Symposium on Theory of Computing, STOC, pages 230-240, 1987.

6 Surender Baswana, Telikepalli Kavitha, Kurt Mehlhorn, and Seth Pettie. Additive spanners and (alpha, beta)-spanners. ACM Trans. Algorithms, 7(1):5, 2010.

7 Surender Baswana and Sandeep Sen. A simple and linear time randomized algorithm for computing sparse spanners in weighted graphs. Random Struct. Algorithms, 30(4):532-563, 2007. 
8 Keren Censor-Hillel and Michal Dory. Distributed Spanner Approximation. In ACM Symposium on Principles of Distributed Computing, PODC, pages 139-148, 2018.

9 Keren Censor-Hillel, Bernhard Haeupler, Jonathan A. Kelner, and Petar Maymounkov. Global computation in a poorly connected world: fast rumor spreading with no dependence on conductance. In 44th Symposium on Theory of Computing Conference, STOC, pages 961-970, 2012.

10 Keren Censor-Hillel, Telikepalli Kavitha, Ami Paz, and Amir Yehudayoff. Distributed Construction of Purely Additive Spanners. In 30th International Symposium on Distributed Computing, DISC, pages 129-142, 2016.

11 Keren Censor-Hillel, Ami Paz, and Noam Ravid. The Sparsest Additive Spanner via Multiple Weighted BFS Trees. CoRR, abs/1811.01997, 2018. arXiv:1811.01997.

12 Shiri Chechik. Compact routing schemes with improved stretch. In ACM Symposium on Principles of Distributed Computing, PODC, pages 33-41, 2013.

13 Atish Das Sarma, Stephan Holzer, Liah Kor, Amos Korman, Danupon Nanongkai, Gopal Pandurangan, David Peleg, and Roger Wattenhofer. Distributed Verification and Hardness of Distributed Approximation. SIAM J. Comput., 41(5):1235-1265, 2012.

14 Bilel Derbel and Cyril Gavoille. Fast deterministic distributed algorithms for sparse spanners. Theor. Comput. Sci., 399(1-2):83-100, 2008.

15 Bilel Derbel, Cyril Gavoille, and David Peleg. Deterministic Distributed Construction of Linear Stretch Spanners in Polylogarithmic Time. In 21st International Symposium on Distributed Computing, DISC, pages 179-192, 2007.

16 Bilel Derbel, Cyril Gavoille, David Peleg, and Laurent Viennot. On the locality of distributed sparse spanner construction. In 27th Annual ACM Symposium on Principles of Distributed Computing, PODC, pages 273-282, 2008.

17 Bilel Derbel, Cyril Gavoille, David Peleg, and Laurent Viennot. Local Computation of Nearly Additive Spanners. In International Symposium on Distributed Computing, DISC, pages 176-190, 2009.

18 Devdatt P. Dubhashi, Alessandro Mei, Alessandro Panconesi, Jaikumar Radhakrishnan, and Aravind Srinivasan. Fast distributed algorithms for (weakly) connected dominating sets and linear-size skeletons. J. Comput. Syst. Sci., 71(4):467-479, 2005.

19 Michael Elkin. Computing almost shortest paths. ACM Trans. Algorithms, 1(2):283-323, 2005.

20 Michael Elkin. Distributed exact shortest paths in sublinear time. In ACM SIGACT Symposium on Theory of Computing, STOC, pages 757-770, 2017.

21 Michael Elkin and Ofer Neiman. Hopsets with Constant Hopbound, and Applications to Approximate Shortest Paths. In IEEE 57th Annual Symposium on Foundations of Computer Science, FOCS, pages 128-137, 2016.

22 Michael Elkin and Jian Zhang. Efficient algorithms for constructing $(1+\epsilon, \beta)$-spanners in the distributed and streaming models. Distributed Computing, 18(5):375-385, 2006.

23 Robert G. Gallager, Pierre A. Humblet, and Philip M. Spira. A Distributed Algorithm for Minimum-Weight Spanning Trees. ACM Trans. Program. Lang. Syst., 5(1):66-77, 1983.

24 Mohsen Ghaffari and Fabian Kuhn. Derandomizing Distributed Algorithms with Small Messages: Spanners and Dominating Set. In International Symposium on Distributed Computing, DISC, volume 121 of LIPIcs, pages 29:1-29:17, 2018.

25 Mohsen Ghaffari and Jason Li. Improved distributed algorithms for exact shortest paths. In SIGACT Symposium on Theory of Computing, STOC, pages 431-444, 2018.

26 Ofer Grossman and Merav Parter. Improved Deterministic Distributed Construction of Spanners. In 31st International Symposium on Distributed Computing, DISC, pages 24:1$24: 16,2017$. 
27 Monika Henzinger, Sebastian Krinninger, and Danupon Nanongkai. A deterministic almost-tight distributed algorithm for approximating single-source shortest paths. In $A C M$ SIGACT Symposium on Theory of Computing, STOC, pages 489-498, 2016.

28 Stephan Holzer. Personal communication.

29 Stephan Holzer, David Peleg, Liam Roditty, and Roger Wattenhofer. Distributed 3/2Approximation of the Diameter. In 28th International Symposium on Distributed Computing, DISC, pages 562-564, 2014.

30 Stephan Holzer and Roger Wattenhofer. Optimal distributed all pairs shortest paths and applications. In ACM Symposium on Principles of Distributed Computing, PODC, pages 355-364, 2012.

31 Chien-Chung Huang, Danupon Nanongkai, and Thatchaphol Saranurak. Distributed Exact Weighted All-Pairs Shortest Paths in O $\left(n^{5 / 4}\right)$ Rounds. In 58th IEEE Annual Symposium on Foundations of Computer Science, FOCS, pages 168-179, 2017.

32 Telikepalli Kavitha. New Pairwise Spanners. In 32nd International Symposium on Theoretical Aspects of Computer Science, STACS, pages 513-526, 2015.

33 Mathias Bæk Tejs Knudsen. Additive Spanners: A Simple Construction. In 14th Scandinavian Symposium and Workshops on Algorithm Theory, SWAT, pages 277-281, 2014.

34 Christoph Lenzen, Boaz Patt-Shamir, and David Peleg. Distributed distance computation and routing with small messages. Distributed Computing, pages 1-25, 2018.

35 Christoph Lenzen and David Peleg. Efficient distributed source detection with limited bandwidth. In ACM Symposium on Principles of Distributed Computing, PODC, pages 375-382, 2013.

36 Zvi Lotker, Boaz Patt-Shamir, and Seth Pettie. Improved Distributed Approximate Matching. J. ACM, 62(5):38:1-38:17, 2015.

37 Danupon Nanongkai. Distributed approximation algorithms for weighted shortest paths. In Symposium on Theory of Computing, STOC, pages 565-573, 2014.

38 Merav Parter. Vertex fault tolerant additive spanners. Distributed Computing, 30(5):357$372,2017$.

39 Merav Parter and Eylon Yogev. Congested Clique Algorithms for Graph Spanners. In International Symposium on Distributed Computing, DISC, volume 121 of LIPIcs, pages 40:1-40:18, 2018.

40 David Peleg. Distributed Computing: A Locality-Sensitive Approach. Monographs on Discrete Mathematics and Applications. Society for Industrial and Applied Mathematics, 2000.

41 David Peleg and Alejandro A. Schäffer. Graph spanners. Journal of Graph Theory, 13(1):99$116,1989$.

42 David Peleg and Jeffrey D. Ullman. An Optimal Synchronizer for the Hypercube. SIAM J. Comput., 18(4):740-747, 1989.

43 David Peleg and Eli Upfal. A trade-off between space and efficiency for routing tables. $J$. ACM, 36(3):510-530, 1989.

44 Seth Pettie. Distributed algorithms for ultrasparse spanners and linear size skeletons. Distributed Computing, 22(3):147-166, 2010.

45 Mikkel Thorup and Uri Zwick. Compact routing schemes. In ACM Symposium on Parallelism in Algorithms and Architectures, SPAA, pages 1-10, 2001.

46 David P. Woodruff. Additive Spanners in Nearly Quadratic Time. In 37th International Colloquium on Automata, Languages and Programming, ICALP, pages 463-474, 2010. 